Supplement of Nat. Hazards Earth Syst. Sci., 22, 117-138, 2022

https://doi.org/10.5194/nhess-22-117-2022-supplement

(c) Author(s) 2022. CC BY 4.0 License.

(c) (i)

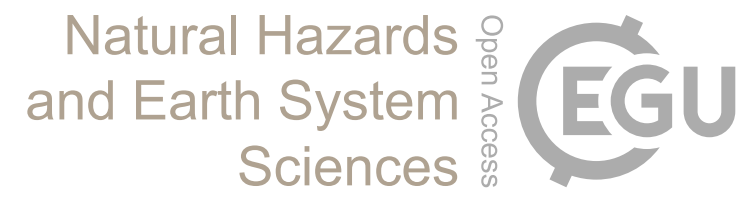

Supplement of

\title{
A sanity check for earthquake recurrence models used in PSHA of slowly deforming regions: the case of SW Iberia
}

Margarida Ramalho et al.

Correspondence to: M. Ramalho (margarida_ramalho@ hotmail.com)

The copyright of individual parts of the supplement might differ from the article licence. 


\section{SUPPLEMENT}

\section{S1. Simple consistency test}

The area of the fault, $A$ (Fig. 5 from the main document) is determined by its length $L$, the thickness of the seismogenic layer

$5 \quad H$ and the dip of the fault $\alpha$, by

$A=\frac{L H}{\sin \alpha}$

To apply the simplified test, we consider that the earthquake recurrence models are expressed in moment magnitude, $m_{W}$ and use the Hanks and Kanamori's law (1979) to convert magnitude into seismic moment

$M_{o}=10^{1.5 m_{w}+9.1}$

10 The velocity along the fault obtained from Eq. (3) from the main document must be converted to horizontal velocity by

$\dot{u}_{h}=\dot{u} \sqrt{(\cos \lambda)^{2}+(\sin \lambda \cos \alpha)^{2}}$

In the simple test applied in this work, we consider that all seismicity occurs in pure thrust faults, for all generation zones, in which case one has $u_{h}=u \cos \alpha$. In this approximation, the most favorable fault plane dip for thrust faults is $35^{\circ}$ for the seismogenic zone likely to have generated the 1755 earthquake (e.g. Sørensen et al., 2012), and $55^{\circ}$ for the Lower Tagus

15 Valley, corresponding to the Continental Rift Boundary class of the work of Bird and Kagan (2004). However, this $55^{\circ}$ and $35^{\circ}$ dip difference is not relevant since $\sin 35 \cos 35=\sin 55 \cos 55=0.47$,

Thus, the slip rate on the fault due to the accumulated seismic activity, $\Delta \dot{u}_{s}$, is given by

$\Delta \dot{u}_{s}=\frac{\sum M_{i}}{\mu L H T} \sin 35^{\circ} \cos 35^{\circ} \rightarrow \Delta \dot{u}_{s} \approx \frac{1}{2} \frac{\sum M_{i}}{\mu L H T}$

\section{S2. Complex sanity test: Seismicity Rates with "Long Term Seismicity"}

Each triangular finite element is subject to horizontal strain rate along the longitude and latitude coordinate system $(\phi, \theta)$ that is computed from the velocity on its nodes. The vertical strain rate, $\dot{\varepsilon}_{r r}$, is then computed invoking incompressibility

$\dot{\varepsilon}_{\phi \phi}+\dot{\varepsilon}_{\theta \theta}+\dot{\varepsilon}_{r r}=0$

Since $\dot{\varepsilon}_{r \phi}$ and $\dot{\varepsilon}_{r \theta}$ should vanish at a shear traction free surface, $\dot{\varepsilon}_{r r}$ is a principal strain rate. Then, the two principal strain

25 rates in the horizontal plane $\left(\dot{\varepsilon}_{1 h} \leq \dot{\varepsilon}_{2 h}\right)$, are given by

$\dot{\varepsilon}_{1 h}=\frac{\dot{\varepsilon}_{\phi \phi}+\dot{\varepsilon}_{\theta \theta}}{2}-\sqrt{\dot{\varepsilon}_{\phi \theta}^{2}+\left(\dot{\varepsilon}_{\phi \phi}-\dot{\varepsilon}_{\theta \theta}\right)^{2} / 4}$

$\dot{\varepsilon}_{2 h}=\frac{\dot{\varepsilon}_{\phi \phi}+\dot{\varepsilon}_{\theta \theta}}{2}-\sqrt{\dot{\varepsilon}_{\phi \theta}^{2}+\left(\dot{\varepsilon}_{\phi \phi}-\dot{\varepsilon}_{\theta \theta}\right)^{2} / 4}$

The three principal strain rates are alternately labelled as an ordered triplet

$\dot{\varepsilon_{1}} \leq \dot{\varepsilon_{2}} \leq \dot{\varepsilon_{3}}$

30 The seismic moment release rate per unit area, $\dot{M} / A$, for the finite element of area $\mathrm{A}$, is then computed by

$\frac{\dot{M}}{A}=\langle c z\rangle \mu\left\{\begin{array}{cc}2 \dot{\varepsilon}_{3} ; & \text { if } \dot{\varepsilon}_{2}<0, \text { or } \\ -2 \dot{\varepsilon}_{1} ; & \text { if } \dot{\varepsilon}_{2} \geq 0\end{array}\right.$ 
Here $\mu$ is the average shear modulus and $\langle c z\rangle$ is the coupled thickness, an average of the product of the seismic coupling parameter, $c$, and the depth of the lithosphere above the brittle/ductile transition, $z$. The seismic moment release equation is based on a kinematic model, where the volume preserving deformation is equivalent to slip on many minor virtual faults falling

35 into (up to) two conjugate sets. The more active conjugate fault set bisects the angles between the principal strain rate axes $\pm \hat{\varepsilon}_{1}$ and $\pm \hat{\varepsilon}_{3}$; the less active conjugate fault set bisects the angles between principal strain rate axes $\pm \hat{\varepsilon}_{2}$ and $\pm \hat{\varepsilon}_{3}$ (if $\dot{\varepsilon}_{2}<0$ ) or between $\pm \hat{\varepsilon}_{1}$ and $\pm \hat{\varepsilon}_{2}$ (if $\dot{\varepsilon}_{2} \geq 0$ ). The factor of \pm 2 appearing in Eq. (S8) is the smallest coefficient possible and comes from the assumption that the virtual fault planes make angles of $45^{\circ}$ with the principal strain rate axes (see Appendix of Carafa et al., 2017). This formula also assumes that the strain rates are long-term permanent strain rates, and not elastic (Bird et al.,

40 2010).

When lithospheric deformation is concentrated along fault planes with long-term average slip rate, $\dot{s}$, then, according to Bird and Liu (2007), the long-term average seismic moment rate on that fault is given by

$\dot{M}=\iint c \mu \dot{s} d a$

Where $c$ is the dimensionless seismic coupling, $d a$ is an element of fault area, and the integral is over the frictional (potentially

45 seismogenic) portion of the fault surface that lies above the brittle/ductile transition. For large blocks of lithosphere, which do not rotate about horizontal axes (although they may rotate about vertical axes), slip rates hardly vary in the down-dip direction, allowing for the following approximation (ibid.)

$\dot{M} \cong\langle c z\rangle \int \mu \sqrt{v_{p}^{2}+\left(v_{o} \sec (\alpha)\right)^{2}} \csc (\alpha) d l$

Here $v_{p}$ is the trace-parallel component of the horizontal relative block velocity vector, $v_{o}$ is the orthogonal (trace-normal)

50 component of the horizontal relative block velocity vector, $\alpha$ is the dip of the fault and $d l$ is a small step along the length of the fault. The integral is taken on the surface along the trace of the fault. We remark that in both cases, seismic strain from diffuse or concentrated deformation, one key parameter is the "mean coupled seismogenic thickness", $\langle c z\rangle$. Only the product is relevant in most computations.

\section{S3. Truncated and Tapered Gutenberg-Richter Distributions}

In order to apply the complex consistency test to the PSHA recurrence models investigated we must first obtain the earthquake recurrence parameters with the Tapered Gutenberg-Richter law ( $\dot{N}^{\text {Comp }}, M_{T}, \beta_{t G R}$ and $\left.m_{c}\right)$, Eq. (S11) below, equivalent to those used with the double truncated Gutenberg-Richter law, Eq. (S12) below.

$\dot{N}\left(m_{T}\right)=\dot{N}^{\operatorname{Comp}}\left(\frac{M_{T}}{M_{C}}\right)^{-\beta_{t G R}} e^{\left(\frac{M^{\operatorname{Comp}}-M_{T}}{M_{C}}\right)}$

$60 \dot{N}(m)=\lambda \frac{e^{-\beta\left(m-m_{\min }\right)}-e^{-\beta\left(m_{\max }-m_{\min }\right)}}{1-e^{-\beta\left(m_{\max }-m_{\min }\right)}}$

In this supplement, we will see in detail, the conversion process. In Tables S1 and S2 we present the expected number of earthquakes in 100 years for different classes of magnitude, between 5.0 and the maximum magnitude of each zone, every 0.5 . The small differences observed are justified by the adjustment of the two laws, one is expressed by magnitude, other by seismic moment, where it was given priority to the equality between seismic moment release rates calculated by the two laws. Since

65 the laws have slightly different algebraic forms, especially at higher magnitudes, differences in the number of earthquakes may occur, which we consider not relevant (in the adjustment made it was convenient that both laws translate the same total sum of the released seismic moment per year so that the total number of earthquakes in each zone would not be affected). The seismic moment release rate $\left(\dot{M}_{0}\right)$ for 100 years is also shown in these tables. 
The Fig. S1 and S2 show the comparison of the two sets of laws for the Lower Tagus Valley and 1755 source zone respectively.

70
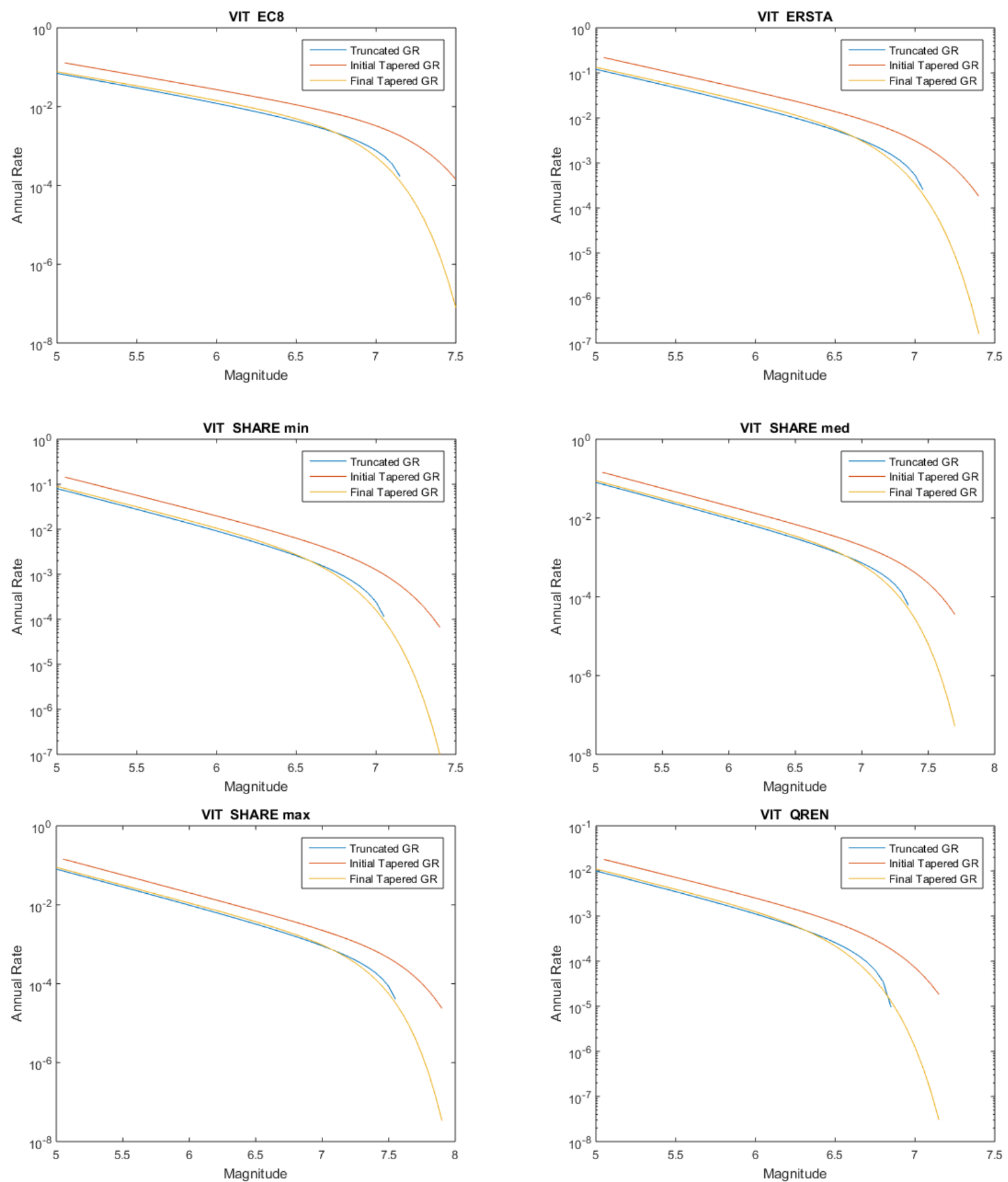

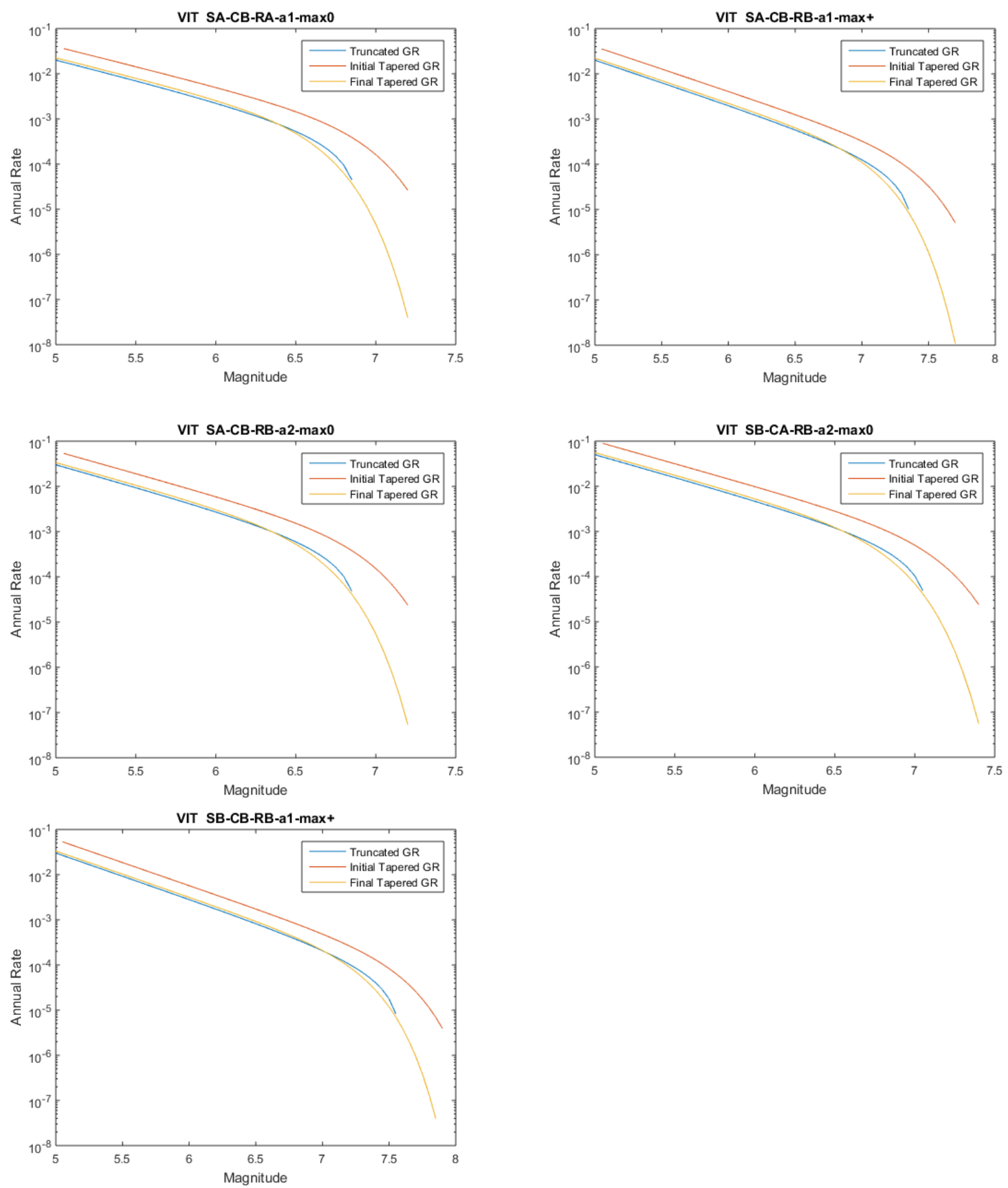

80 Fig. S1: (on this and previous page) Comparison between the truncated Gutenberg-Richter law (blue) and initial Tapered Gutenberg-Richter law (orange) and final TGR (yellow), for LTV source zone considering the referred 11 recurrence models investigated with the complex sanity test. 

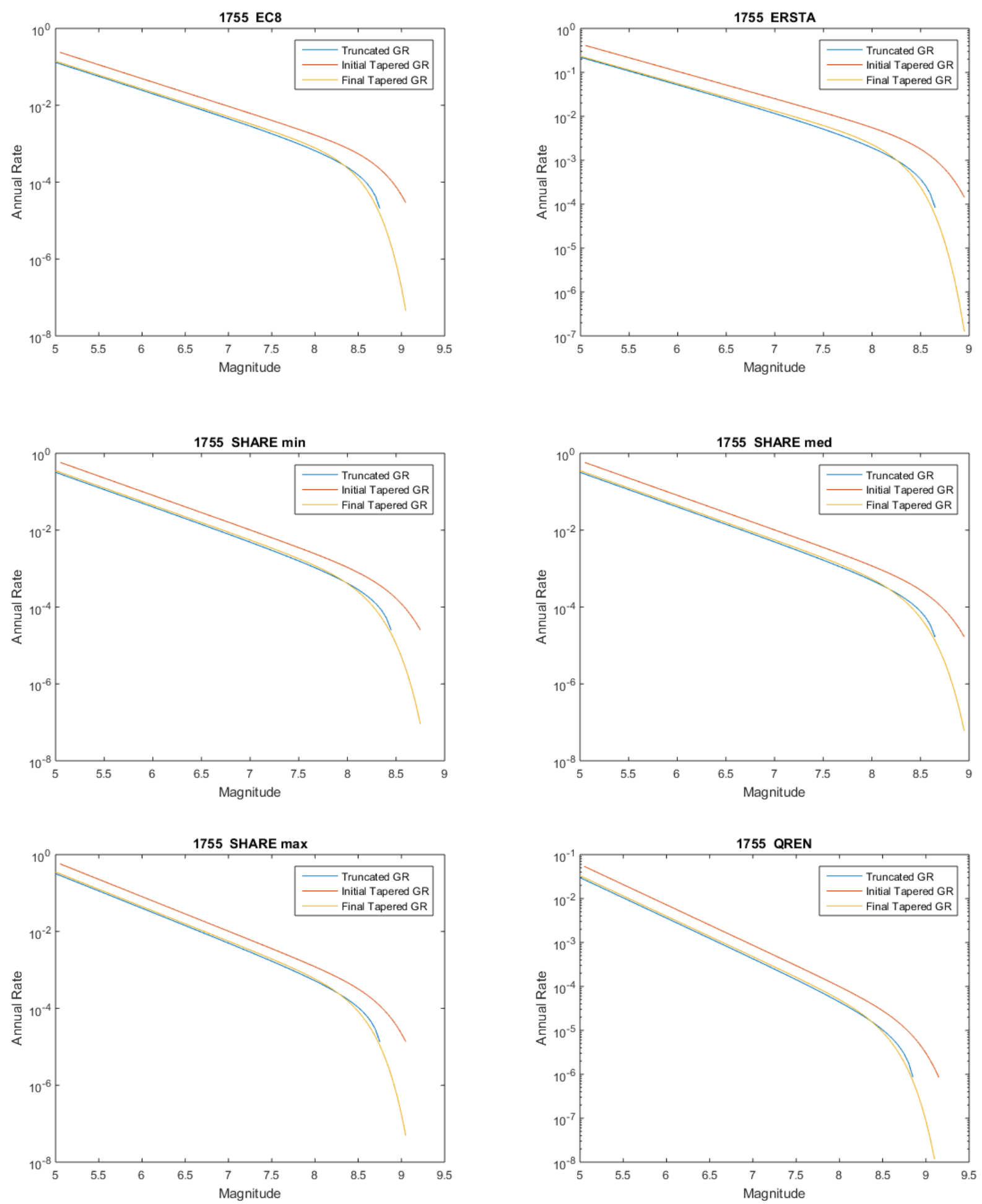

85 

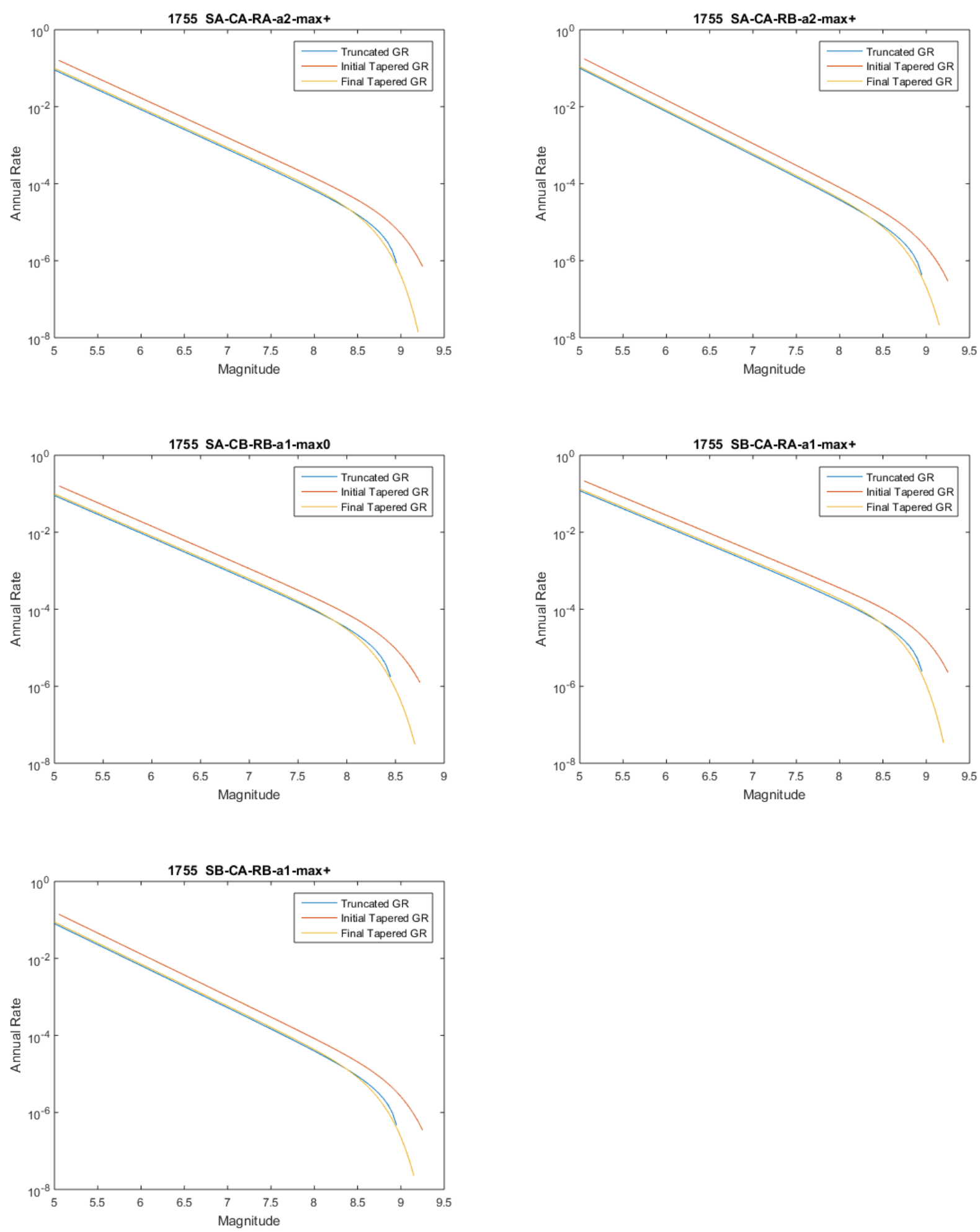

90

Fig. S2: (on this and previous page) Comparison between the truncated Gutenberg-Richter law (blue) and initial "tapered" GutenbergRichter law (orange) and final "tapered" (yellow), for 1755 source zone considering the referred 5 proposals. 
Table S1: Activity rates in EQs/century for the Lower Tagus Valley zone for each model, for magnitude 5.0 to 7.0, every 0.5. It is presented, for 100 years, the total seismic moment release rate. In blue the calculus with the truncated Gutenberg-Richter law and black the "tapered" Gutenberg-Richter law, for 100 years of catalogue duration. $\dot{M}_{0}$ as the long-term seismic moment rate.

\begin{tabular}{|c|c|c|c|c|c|c|}
\hline \multicolumn{7}{|c|}{ Lower Tagus Valley (LTV) } \\
\hline \multirow{2}{*}{ Model } & \multicolumn{5}{|c|}{ Magnitude } & \multirow{2}{*}{$\begin{array}{c}\dot{M}_{o} \\
(\mathrm{Nm} / \text { century })\end{array}$} \\
\hline & 5.0 & 5.5 & 6.0 & 6.5 & 7.0 & \\
\hline \multirow{3}{*}{ EC8 } & 7.0 & 2.99 & 1.21 & 0.425 & 0.077 & \multirow{3}{*}{$1.29 \times 10^{19}$} \\
\hline & & & & & & \\
\hline & 8.6 & 3.77 & 1.58 & 0.511 & 0.039 & \\
\hline \multirow{3}{*}{ ERSTA } & 12.0 & 4.67 & 1.72 & 0.532 & 0.054 & \multirow{3}{*}{$1.50 \times 10^{19}$} \\
\hline & & & & & & \\
\hline & 14.9 & 5.92 & 2.21 & 0.580 & 0.021 & \\
\hline \multirow{3}{*}{ SHARE $_{\min }$} & 8.0 & 2.77 & 0.92 & 0.258 & 0.024 & \multirow{3}{*}{$7.65 \times 10^{18}$} \\
\hline & & & & & & \\
\hline & 9.8 & 3.47 & 1.15 & 0.274 & 0.010 & \\
\hline \multirow{3}{*}{ SHARE $_{\text {med }}$} & 8.0 & 2.81 & 0.96 & 0.305 & 0.072 & \multirow{3}{*}{$1.17 \times 10^{19}$} \\
\hline & & & & & & \\
\hline & 9.8 & 3.47 & 1.20 & 0.368 & 0.057 & \\
\hline \multirow{3}{*}{ SHARE $_{\max }$} & 8.0 & 2.82 & 0.98 & 0.323 & 0.091 & \multirow{3}{*}{$1.56 \times 10^{19}$} \\
\hline & & & & & & \\
\hline & 9.8 & 3.47 & 1.22 & 0.402 & 0.095 & \\
\hline \multirow{3}{*}{ QREN } & 1.0 & 0.35 & 0.11 & 0.026 & --- & \multirow{3}{*}{$6.91 \times 10^{17}$} \\
\hline & & & & & & \\
\hline & 1.2 & 0.42 & 0.13 & 0.019 & --- & \\
\hline \multirow{2}{*}{$\begin{array}{l}\text { SA-CB-RA-a1- } \\
\max 0\end{array}$} & 2.0 & 0.69 & 0.22 & 0.053 & --- & \multirow{2}{*}{$1.46 \times 10^{18}$} \\
\hline & 2.2 & 0.77 & 0.25 & 0.047 & --- & \\
\hline \multirow{2}{*}{$\begin{array}{c}\text { SA-CB-RB-a1- } \\
\max +\end{array}$} & 2.0 & 0.63 & 0.20 & 0.057 & 0.013 & \multirow{2}{*}{$2.23 \times 10^{18}$} \\
\hline & 2.2 & 0.70 & 0.22 & 0.063 & 0.011 & \\
\hline \multirow{2}{*}{$\begin{array}{l}\text { SA-CB-RB-a2- } \\
\max 0\end{array}$} & 3.0 & 0.93 & 0.27 & 0.060 & --- & \multirow{2}{*}{$1.79 \times 10^{18}$} \\
\hline & 3.4 & 1.07 & 0.31 & 0.052 & --- & \\
\hline \multirow{2}{*}{$\begin{array}{l}\text { SB-CB-RB-a1- } \\
\max +\end{array}$} & 3.0 & 0.92 & 0.28 & 0.082 & 0.021 & \multirow{2}{*}{$3.86 \times 10^{18}$} \\
\hline & 3.3 & 1.02 & 0.31 & 0.091 & 0.021 & \\
\hline \multirow{2}{*}{$\begin{array}{l}\text { SB-CA-RB-a2- } \\
\max 0\end{array}$} & 5.0 & 1.56 & 0.47 & 0.12 & 0.010 & \multirow{2}{*}{$3.76 \times 10^{18}$} \\
\hline & 5.6 & 1.76 & 0.53 & 0.12 & 0.007 & \\
\hline
\end{tabular}

The seismic moment release rates are refereed to magnitude 5.0. 
Table S2: Activity rates in EQs/century for the 1755 source zone for each model, for magnitude 5.0 to 7.0, every 0.5. It is presented, for 100 years, the total seismic moment release rate. In blue the calculus with the Double Truncated Gutenberg-Richter law and black the Tapered Gutenberg-Richter law, for 100 years of catalogue duration. $\dot{M}_{0}$ as the long-term seismic moment rate.

\begin{tabular}{|c|c|c|c|c|c|c|c|c|c|}
\hline \multicolumn{10}{|c|}{1755 Source zone } \\
\hline \multirow{2}{*}{ Model } & \multicolumn{8}{|c|}{ Magnitude } & \multirow[t]{2}{*}{$\begin{array}{l}\dot{M}_{o}(\mathrm{Nm} / \\
\text { century })\end{array}$} \\
\hline & 5.0 & 5.5 & 6.0 & 6.5 & 7.0 & 7.5 & 8.0 & 8.5 & \\
\hline \multirow[t]{2}{*}{ EC8 } & 13.0 & 5.66 & 2.45 & 1.06 & 0.45 & 0.18 & 0.066 & 0.015 & \multirow[t]{2}{*}{$4.04 \times 10^{2}$} \\
\hline & 15.7 & 6.85 & 2.98 & 1.30 & 0.563 & 0.236 & 0.082 & 0.010 & \\
\hline \multirow{3}{*}{ ERSTA } & 22.0 & 10.7 & 5.18 & 2.48 & 1.15 & 0.508 & 0.192 & 0.037 & \multirow{3}{*}{$1.03 \times 10^{21}$} \\
\hline & & & & & & & & & \\
\hline & 26.2 & 12.8 & 6.27 & 3.06 & 1.48 & 0.683 & 0.239 & 0.018 & \\
\hline \multirow{3}{*}{ SHARE $_{\min }$} & 32.0 & 11.4 & 4.02 & 1.41 & 0.487 & 0.158 & 0.042 & 0 & \multirow{3}{*}{$2.20 \times 10^{20}$} \\
\hline & & & & & & & & & \\
\hline & 38.8 & 13.8 & 4.89 & 1.732 & 0.604 & 0.194 & 0.038 & $5.2 \times 10^{-4}$ & \\
\hline \multirow{3}{*}{ SHARE $_{\text {med }}$} & 32.0 & 11.4 & 4.02 & 1.42 & 0.495 & 0.166 & 0.049 & 0.008 & \multirow{3}{*}{$2.90 \times 10^{2}$} \\
\hline & & & & & & & & & \\
\hline & 38.8 & 13.8 & 4.89 & 1.736 & 0.611 & 0.206 & 0.055 & 0.004 & \\
\hline \multirow{3}{*}{ SHARE $_{\max }$} & 32.0 & 11.4 & 4.03 & 1.42 & 0.497 & 0.169 & 0.052 & 0.011 & \multirow{3}{*}{$3.33 \times 10^{20}$} \\
\hline & & & & & & & & & \\
\hline & 38.8 & 13.8 & 4.89 & 1.737 & 0.613 & 0.230 & 0.061 & 0.007 & \\
\hline \multirow{3}{*}{ QREN } & 3.0 & 1.04 & 0.36 & 0.12 & 0.043 & 0.014 & 0.004 & 0.001 & \multirow{3}{*}{$3.13 \times 10^{19}$} \\
\hline & & & & & & & & & \\
\hline & 3.6 & 1.25 & 0.43 & 0.15 & 0.052 & 0.017 & 0.005 & $8.2 \times 10^{-4}$ & \\
\hline \multirow{2}{*}{$\begin{array}{l}\text { SA-CB-RB-a1- } \\
\max 0\end{array}$} & 9.0 & 2.54 & 0.72 & 0.20 & 0.056 & 0.015 & 0.003 & 0 & \multirow{2}{*}{$2.17 \times 10^{1}$} \\
\hline & 9.9 & 2.79 & 0.79 & 0.22 & 0.062 & 0.016 & 0.003 & $8.0 \times 10^{-5}$ & \\
\hline \multirow{2}{*}{$\begin{array}{c}\text { SB-CA-RB-a1- } \\
\text { max+ }\end{array}$} & 8.0 & 2.28 & 0.65 & 0.185 & 0.053 & 0.015 & 0.004 & $9.0 \times 10^{-4}$ & \multirow{2}{*}{$3.27 \times 10^{1}$} \\
\hline & 8.8 & 2.51 & 0.72 & 0.204 & 0.058 & 0.016 & 0.004 & $8.2 \times 10^{-4}$ & \\
\hline \multirow{2}{*}{$\begin{array}{c}\text { SA-CA-RB-a2- } \\
\text { max+ }\end{array}$} & 10.0 & 2.7 & 0.74 & 0.20 & 0.055 & 0.015 & 0.004 & $8.0 \times 10^{-4}$ & \multirow{2}{*}{$3.22 \times 10^{19}$} \\
\hline & 10.9 & 3.0 & 0.810 & 0.221 & 0.060 & 0.016 & 0.004 & $7.4 \times 10^{-4}$ & \\
\hline \multirow{2}{*}{$\begin{array}{c}\text { SA-CA-RA-a2- } \\
\max +\end{array}$} & 9.0 & 2.75 & 0.84 & 0.257 & 0.078 & 0.023 & 0.002 & 0 & \multirow{2}{*}{$5.36 \times 10^{19}$} \\
\hline & 9.9 & 3.03 & 0.93 & 0.283 & 0.086 & 0.026 & 0.007 & $1.47 \times 10^{-3}$ & \\
\hline \multirow{2}{*}{$\begin{array}{c}\text { SB-CA-RA-a1- } \\
\max +\end{array}$} & 12.0 & 4.07 & 1.38 & 0.47 & 0.16 & 0.052 & 0.016 & 0.004 & \multirow{2}{*}{$1.28 \times 10^{20}$} \\
\hline & 13.3 & 4.52 & 1.534 & 0.521 & 0.176 & 0.059 & 0.019 & $4.05 \times 10^{-3}$ & \\
\hline
\end{tabular}

The seismic moment release rates are refereed to magnitude 5.0.

When comparing the earthquake rates between DTGR and TGR we note, for both source zones and all recurrence models, that the number of earthquakes is higher for the TGR on all classes of magnitude, except for the largest maximum magnitude evaluated for each zone. However, the seismic moment release rates are identical on both DTGR and TGR recurrence laws. 


\section{References}

Bird, P. and Kagan, Y. Y.: Plate-tectonic analysis of shallow seismicity: Apparent boundary width, beta, corner magnitude, coupled lithosphere thickness, and coupling in seven tectonic settings, B. Seismol. Soc. Am., 94, 2380-2399, 2004.

Bird, P. and Liu, Z.: Seismic hazard inferred from tectonics: California, Seismol. Res. Lett., 78, 37-48, 2007.

Bird, P., Kreemer, C., and Holt,W. E.: A long-term forecast of shallow seismicity based on the Global Strain Rate Map, 115 Seismol. Res. Lett., 81, 184-194, 2010.

Carafa, M. M., Valensise, G., and Bird, P.: Assessing the seismic coupling of shallow continental faults and its impact on seismic hazard estimates: a case-study from Italy, Geophys. J. Int., 209, 32-47, 2017.

Hanks, T. C. and Kanamori, H.: A moment magnitude scale, J. Geophys. Res., 84, 2348-2350, 1979.

Sørensen, M. B., Spada, M., Babeyko, A., Wiemer, S., and Grünthal, G.: Probabilistic tsunami hazard in the Mediterranean 